\title{
Synthesis of multidimensional pathophysiological process leading to type A aortic dissection: a narrative review
}

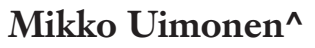 \\ Department of Surgery, Central Finland Hospital Nova, Jyväskylä, Finland \\ Correspondence to: Mikko Uimonen. Department of Surgery, Central Finland Hospital Nova, Hoitajantie 3, 40620, Jyväskylä, Finland. \\ Email: mikko.m.uimonen@gmail.com.
}

Objective: This review aims to synthesize the existing knowledge on the etiological process leading to type A aortic dissection (TAAD) and to clarify the relationship between mechanical, biochemical, and histopathological processes behind the aortic disease.

Background: Extensive research has previously identified several risk factors for TAAD as well as pathological mechanisms leading to TAAD. However, due to the complexity of the pathological process and limited knowledge on the relationships between distinct pathomechanisms leading to TAAD, the ability to identify the patients at high risk for TAAD has been poor.

Methods: PubMed (National Library of Medicine) database was searched for suitable literature. The most relevant articles focusing on anatomy, histopathology, physiology, and mechanics of ascending aorta and aortic diseases were reviewed.

Conclusions: Pathophysiology of the TAAD is related to biochemical and histological as well as mechanical and hemodynamic alterations leading to a degeneration of the aortic wall via inflammatory response. The degradative mechanisms of aortic wall structures and the mechanical forces, to which the wall is predisposed, are interrelated and influence one another. The relativity between the factors influencing aortic wall strength and healing capacity, and factors influencing mechanical stress on the aortic wall suggest that the risk of TAAD is not a linear but rather a dynamic phenomenon. Accounting for the dynamical property of the aortic disease in assessing the need for preventive surgical aortic reconstruction may provide a wider perspective in identifying patients at risk of TAAD and in planning preventive medical therapies.

Keywords: Type A aortic dissection (TAAD); histopathology; pathophysiology; biomechanics

Submitted May 13, 2021. Accepted for publication Aug 06, 2021.

doi: $10.21037 /$ jtd-21-829

View this article at: https://dx.doi.org/10.21037/jtd-21-829

\section{Introduction}

Dissection of ascending aorta, type A aortic dissection (TAAD), is a vascular catastrophe that is related to high mortality without emergent surgery (1-3). Dissection occurs when blood infiltrates between the layers of the aortic wall through a tear in the intima, the innermost layer of the aorta (1). Blood flow detaches the aortic layers from each other along the vessel and forms a false lumen between the aortic layers (1). The false lumen is separated from the true aortic lumen by intima. Complications of TAAD, such as cardiac tamponade, thromboembolic events, and end-organ malperfusion due to disrupted blood flow in branching vessels, are devastating and potentially lethal (1). The emergency surgery aims to secure blood flow in the true lumen of the aorta and the branching vessels by replacing the diseased aortic section with a prosthesis.

\footnotetext{
^ ORCID: 0000-0001-6609-1345.
} 


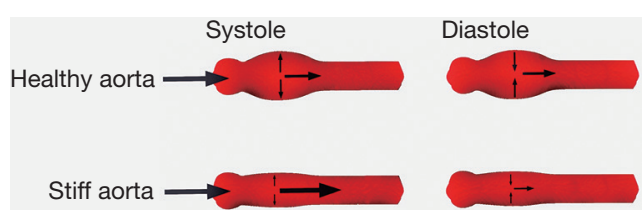

Figure 1 Schematic illustration of the Windkessel effect in ascending aorta. Systolic increase in intraluminal blood pressure causes tension in the compliant ascending aorta wall. In diastole when the pressure normalizes, the tension is released maintaining blood flow also during diastole. Stiffening of ascending aorta due to degenerative process weakens the Windkessel effect leading to higher systolic and lower diastolic blood pressure. The arrows represent blood flow from the left heart ventricle to ascending aorta and the size of the arrow shows the flow speed.

Previous research has identified several risk factors for TAAD. Likely the most evident predictive risk factors identified in previous research have been connective tissue disorders, pre-existing aneurysm in ascending aorta, blunt trauma on the chest, or iatrogenic dissection (4). However, a large proportion of TAAD patients have no such exposures. In contrast, a higher burden of cardiovascular risk factors and diseases have been found associated with the risk of TAAD (4). Nonetheless, the ability to identify the patients at high risk for TAAD has been limited and a majority of TAAD cases have remained unpredictable thus far. To patch this flaw, extensive research during past decades has shed a light on the complexity and multidimensionality of the underlying pathological process of TAAD. Mechanical, biochemical, and histopathological studies have increased knowledge on the natural history of aortic disease ultimately leading to TAAD.

This review aims to synthesize the existing knowledge on the etiological process leading to TAAD and to clarify the relationship between mechanical, biochemical, and histopathological processes behind the aortic disease.

I present the following article in accordance with the Narrative Review reporting checklist (available at https:// dx.doi.org/10.21037/jtd-21-829).

\section{Methods}

PubMed (National Library of Medicine) database was searched for suitable literature. Literature search was conducted using terms "aortic dissection", "aortic dilatation" or "aortic aneurysm" combined with terms "anatomy", "physiology", "histology", "biochemistry", "biomechanics", "inflammation", "risk factors", "fluid dynamics" or "hemodynamics". Only published articles written in English were considered. The most relevant articles focusing on anatomy, histopathology, physiology, and mechanics of ascending aorta and aortic diseases were reviewed.

\section{Anatomy, physiology, and mechanics of healthy ascending aorta}

\section{Functional anatomy}

The main function of the aorta is mechanical involving the dissemination of blood flow to the branching vessels. The ascending portion of the aorta, comprising aortic root and ascending tubular aorta extending to the brachiocephalic trunk, carries the highest stress during the cardiac cycle $(5,6)$. A healthy ascending aorta is compliant to the intraluminal pressure variation to decrease blood flow impedance $(6,7)$. During systole, blood flow from the left cardiac ventricle through the aortic valve to ascending aorta increases pressure in the aortic lumen. Owing to the histological structure of the media layer, the aortic wall distally to sinotubular junction is compliant to increased pressure leading to distension of aortic wall during systole and releasing during diastole when the pressure normalizes (6-8). In other words, during systole when the pressure in the aortic lumen increases, the kinetic energy of blood flow is transformed into potential energy of elastic wall, whereas during diastole, potential energy stored in the elastic aortic wall transforms into kinetic energy of intraluminal blood (Figure 1). Compliance of the wall of ascending aorta during the cardiac cycle, the so-called Windkessel effect, decreases systolic blood flow impedance and is important in protecting smaller arteries from the systolic increase in blood pressure as well as in maintaining organ perfusion during diastole (6-8).

With a normal aortic valve and ascending aorta, systolic transvalvular blood flow jet through the aortic valve is parallel to the ascending aorta with only minor maldistributions in stress towards the aortic wall (Figure 2) (9). To enhance the blood flow in physiological conditions, the angle between the heart and ascending aorta decreases during systole straightening the outflow track and facilitating blood flow in the aortic lumen whereas, during the diastole, the angle increases again recovering to the resting state (10). 


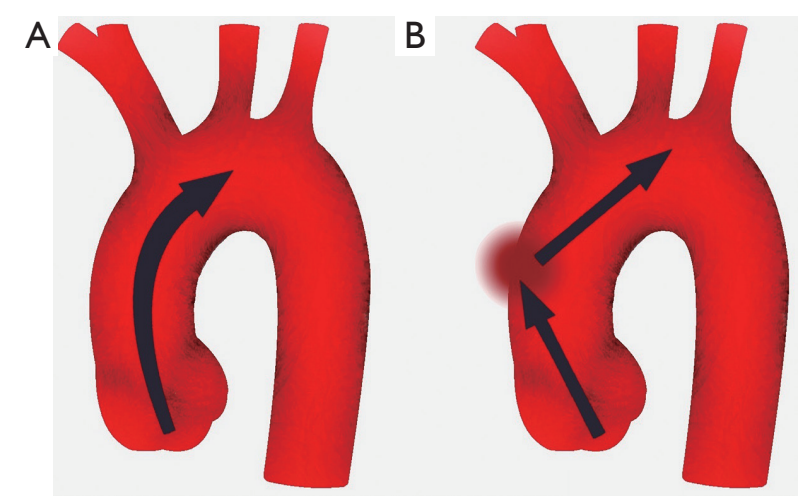

Figure 2 In normal aorta (A), the transvalvular blood flow jet (arrows) is parallel to the aortic lumen. Conditions affecting cardiac outflow track shift the jet direction towards the middle section of ascending aorta convexity (B) causing localized excessive mechanical stress.

\section{Microanatomy and histology}

The aortic wall consists of three layers: the innermost layer intima, the outermost layer adventitia, and media between the two (11). Intima is a thin layer covering the surface of the aortic lumen and consists of an endothelial cell layer and a basal membrane. The thickest layer media consists of smooth muscle cells and connective tissue fibers, especially elastin and collagen as well as glycosaminoglycans and glycoproteins $(11,12)$. Elasticity and compliance to pulsatile blood flow of the aorta are mainly functions of the media layer (11). Due to the high elastin and collagen content of the media layer of ascending aorta, the thickness of the aortic wall is highest in the proximal aorta enabling the highest compliance and elasticity in the ascending aorta while decreasing distally (11). Adventitia is a fibrous layer consisting mostly of collagen and a small blood vessel network, vasa vasorum, supplying blood to the outer aortic wall (13). In unison with the aortic thickness, the density of vasa vasorum is highest in the proximal aorta and decreases distally $(13,14)$. Vasa vasorum of ascending aorta originates from coronary arteries or brachiocephalic trunk (15). It has been previously believed, that vasa vasorum penetrate only the outer half of the media layer. However, vasa vasorum vessels have been detected also in the innermost quartile of the aortic wall although the density of vasculature decreases towards the aortic lumen $(13,16)$. Since the systolic increase in aortic intraluminal pressure compresses vasa vasorum vessels closed, similarly to coronary arteries, the blood flow of vasa vasorum of ascending aorta occurs mainly during diastole (17). In addition to flow regulation by cardiac cycle, vasa of ascending aorta are capable of self-regulating their blood flow through mechanoreceptors and chemoreceptors reacting to vasoconstrictors and dilatators in blood flow (18). They are also neuronally innervated by mostly sympathetic nerve fibers (18).

\section{Histopathology of aortic wall degeneration}

\section{Triggers of inflammation in the aortic wall}

Extensive research during past decades has shown that the chain of events leading to TAAD through aortic wall degeneration is a complex phenomenon regulated by several molecular, cellular, and mechanical pathways. Inflammation in the aortic wall has been shown to play a key role in the development of degeneration $(12,19)$. Vascular smooth muscle cells (VSMC) in the aortic media layer have been proposed to play a decisive role in inducing inflammatory cascade as a consequence of mechanical or oxidative stress $(12,19,20)$.

Chronic arterial hypertension has consistently been found to be associated with an increased risk of TAAD (21-23). Hypertensive blood pressure and disturbances in intraluminal blood flow in the ascending aorta increase direct mechanical stress of VSMC in the aortic media provoking a proinflammatory response $(20,24,25)$. In addition to the mechanical effect of arterial hypertension on VSMC, hypertension has also an indirect activating effect on medial degeneration via alteration of vasa vasorum blood flow. Increased pressure in the media layer of ascending aorta due to hypertensive blood pressure in the aortic lumen compresses vasa vasorum in the outer media layer compromising the blood flow (18). Since the perfusion of vasa vasorum is focused on diastole in the physiological state, the vasa perfusion is particularly prone to compromise in increased diastolic aortic pressure $(15,18)$.

On the other hand, perfusion self-regulation of vasa vasorum may play a part in the compromised perfusion during arterial hypertension. Mechanoreceptors of precapillary arterioles sense the increased arterial blood pressure and activate contraction of VSMCs of vasa vasorum protecting capillaries from harmful effects of high pressure (18). Chronic hypertension may lead to increased sensitivity of mechanoreceptors and increased responsiveness of VSMCs leading to inadequate arteriole contraction and thus compromised capillary perfusion (18). Altered capillary perfusion in vasa vasorum penetrating the 
media layer increases hypoxic stress of VSMC in the outer media layer of ascending aorta (26).

Further, atherosclerosis of vasa vasorum alters nourishing blood flow in the outer aortic wall. Endothelial dysfunction leads to luminal obstruction by thickening of the intimal layer of vasa vasorum (15). This may lead to decreased blood flow through vasa capillaries as well as to compromised transvascular diffusion (15). Thus, atherosclerosis may predispose the vascularity area of the diseased vessels to impaired metabolism and increased hypoxic stress. In conjunction with alterations in vasa vasorum blood flow in arterial hypertension, atherosclerotic lesions may intensify the hypoxia in the aortic media.

Smoking has been shown to intensify the inflammatory stimulus within the aortic wall (27). The substances acquired by smoking have been shown to stimulate constriction of arterial walls via decreased sensitivity to nitric oxide and enhance inflammatory response and hypoxic stress in the vessel wall (27). In addition, as a response to sympathetic nervous activation by nicotine, smoking has been shown to increase blood pressure and thus, related effects on the aortic wall (28).

\section{Inflammatory response and media degeneration}

Increased oxidative and mechanical stress of VSMC facilitate the transformation of VSMC phenotype from contractile to synthetic (29). In the synthetic form, as a response to increased stress, VSMCs excrete proinflammatory chemokines to activate and recruit inflammatory cells, especially macrophages, as well as proteolytic enzymes, such as matrix metalloproteases, leading to degradation of the extracellular matrix (ECM) (12). The subsequent cascade causes elastin fragmentation and cleaving of cross-links between the ECM fibers as well as attachments of VSMC to ECM structures (12). Excessive oxidative stress and detachment from ECM fibers provoke an apoptotic stimulus in VSMC (12). Further, fragmented ECM components attract inflammatory cells thereby activating cell-mediated immunological response (12).

As a response to the fragmentation of elastin fibers, the synthesis of collagen is accelerated to correspond to the tension load transferred to collagen fibers from fragmented elastin in the aortic wall $(6,30,31)$. However, the protease imbalance leads to disturbance in collagen synthesis resulting in the formation of uncoiled and irregularly arranged collagen network in outer media and adventitia with deficient strength and decreased compliance of aortic wall (32). Of note, the increased stiffness of the outer aortic wall may further impair the vasa vasorum perfusion due to obstructing effect by intraluminal aortic blood pressure accelerating hypoxemic stress of the media layer. Along with alterations in elastin and collagen compositions, the inflammation has been shown to be related to a localized accumulation of glycosaminoglycans of ECM forming pools in the media layer (12).

Chronic inflammation promotes the production of both pro and antiangiogenic agents in the aortic media (33). These agents activate the formation of new vasa vasorum vessel branches penetrating the media layer from the adventitial vasa network (33). However, inappropriately regulated angiogenesis results in a formation of anomalous neovessels with an incomplete structure characterized by defective junctions between endothelial cells, discontinuous or even absent basal lamina, and deficient smooth muscle cells in the vasa wall (33). Due to the deficits in the wall structure, perfusion through vasa capillaries as well as transfusion through vasa wall are poorly regulated leading to leaky capillaries with increased flow and increased extravasation $(33,34)$. In animal models, hyperglycemia has been found to inhibit the secretion of proangiogenic agents leading to reduced formation of leaky neovessels (35). This may be contributed to the reduced risk of aortic dissection in patients with diabetes although the causality requires further research (36-38).

In a normal aortic wall, the tensile elastin network in ECM maintains the interstitial pressure, a resisting force against excessive fluid diffusion in the interstitial space $(39,40)$. Due to elastic fiber fragmentation, interstitial pressure is decreased allowing increased extravasation of fluid and plasma proteins to interstitial space (40). The resulting edema and swelling of pooled interstitial hydrophilic glycosaminoglycans lead to impaired cohesion of the outer media layer $(34,41)$. The impaired force transmitting properties of degraded elastin and swelled glycosaminoglycans predisposes the aortic wall to localized unequal distribution of stress load during the cardiac cycle $(5,34,41)$. These modifications lead to reductions in the aortic wall strength to resist dissecting.

In connective tissue disorders, the mechanism of inflammatory stimulus and aortic degeneration are somewhat distinct from the presented. Marfan and LoeysDietz syndromes are characterized by the abnormal manifestation of the transforming growth factor-beta (TGF- $\beta$ ) signaling pathway, which plays a key role in activating inflammatory response $(12,19)$. In normal tissue, 
TGF- $\beta$ is bound by fibrillin fibers of ECM and functions in tissue remodeling $(12,19)$. In Marfan patients, the structure of fibrillin is altered impairing the capability to bind TGF- $\beta$ leading to increased TGF- $\beta$ concentration in ECM $(12,19)$. In Loeys-Dietz syndrome, the gene mutations affect the TGF- $\beta$ receptor leading to dysregulated receptor activity $(12,19)$. Either way, increased transcription of genes activated by the TGF- $\beta$ pathway leads to decreased proliferation and increased apoptosis of VSMCs and increased destruction of normal ECM proteins, and increased collagen synthesis $(12,19)$. In Ehlers-Danlos syndrome, the mechanism of aortic degeneration differs from the TGF- $\beta$ pathway mediated degeneration. The vascular type of Ehlers-Danlos syndrome has been found to associate with defective collagen synthesis leading to a weakened aortic wall structure and impaired renewal capacity $(12,19)$.

\section{Mechanical stress and hemodynamic characteristics of ascending aorta}

\section{Altering mechanical properties of the aortic wall}

The ascending aorta has been shown to conform to the intraluminal hemodynamics throughout life $(7,42)$. However, cardiovascular degeneration increases by aging (43-45). The cumulative vascular degeneration leads to attenuation of aortic conformation of the aortic wall around 60 years of age (46-49). Thereafter, the aortic wall becomes more susceptible to accelerated degeneration when predisposed to excessive mechanical and oxidative stress (46-49).

Along with the age-related degenerative process, stiffness of the aortic wall increases and compliance to changing pressure during the cardiac cycle decreases in a gradual process, which is probably related to similar mechanisms as described earlier in this review $(12,19,48,49)$. In addition, increased diastolic aortic pressure in hypertensive patients and subsequently increased wall tension reduces the reserve of the aorta to respond to the systolic increase in blood pressure (50). Decreased aortic wall compliance impairs the transforming of the kinetic energy of flowing blood into potential energy during systole and vice versa during diastole by the elastic wall (8). This leads to increased absorbance of energy in the wall and thereby damaging the wall structure and accelerated degeneration $(24,25)$. Indeed, increased stiffness and reduced responsiveness of the aortic wall to systolic increase in blood pressure have been reported to be related to accelerated aortic dilatation (8).

\section{Intraluminal blood flow dynamics}

In an ideal condition, the transvalvular blood flow jet from the left ventricle is parallel to the lumen of ascending aorta with only minimal turbulence (9). However, due to anatomical properties of the cardiac outflow tract and ascending aorta, the jet direction does not comply with the ideality. The intensity and distribution of stress towards the aortic wall caused by intraluminal hemodynamics during systole are related to aortic shape, the angle between outflow tract from the left ventricle to ascending aorta, diameter, and shape of aortic orifice, and aortic wall compliance (51-57). These factors may alter the speed and direction of the transvalvular blood flow jet from the ideality.

Decreasing compliance of the ascending aorta increases the jet speed in the aortic lumen (Figure 2) (56,57). In patients with more prominent angulation between the cardiac outflow tract and the lumen of ascending aorta, the direction of the jet is shifted towards the great curvature of ascending aorta causing higher stress in the affected region (55). It has been reported that angulation increases by age resulting in higher aortic wall stress in the aged population $(55,58)$. Likewise, abnormal valvular morphology may shift the jet direction towards the aortic wall $(51,53,59,60)$. In patients with bicuspid aortic valve (BAV), the jet direction has been shown to point towards great curvature in the mid-section of ascending aorta $(51,53)$. Although the results on which BAV configuration is related to the highest wall stress have been contradictory, fluid dynamics simulation studies have shown that all BAV configurations relate to at least some degree of increased wall stress when compared to the tricuspid aortic valve (TAV) $(51,61)$. Further, conditions obstructing the aortic outflow tract, usually related to aortic stenosis, BAV, or aortic coarctation, increase the jet speed increasing shear stress in the aortic wall $(51,61)$. As a result of higher mechanical stress by distorted blood flow jet, the middle section of the ascending aorta convexity is found to be more prone to intimal tear than other regions in the ascending aorta (62). Lastly, fluid dynamic studies have shown that dilatation of ascending aorta increases vortex flow and turbulence and subsequently increases wall stress distally to the dilated aortic section (63).

\section{Interaction between aortic wall and aortic blood flow}

When compared to intima and adventitia, the structure of the media layer involves considerably higher elasticity (5). 
The media layer serves as an elastic cushion connecting the less compliant intima and adventitia layers (5). The impedance of pulsatile blood flow causes a longitudinal force towards the intima (64). The force is transmitted to the adventitia through connective tissue fibers of the media layer maintaining the cohesion of the aortic wall (5). Due to elastic properties, the media layer has been shown to carry the highest burden of the forces directed towards the aortic wall caused by intraluminal hemodynamic disturbances $(5,64)$. However, as a result of progressed aortic media degeneration, involving the fragmentation of elastic fibers, VSMC apoptosis, and interstitial edema, the tensile strength of media decreases and the ability to maintain the cohesion deteriorates, especially in case of insufficient healing capacity due to inflammation-related disturbances in collagen synthesis. This allows the intima and the adventitia layers to move longitudinally more freely in relation to each other by the pulsatile blood flow (65). In addition, along with the decreased ability of media to transmit the kinetic energy of pulsatile blood flow to outer aortic layers, intima is exposed to increased stress, which predisposes it to tear $(30,64,65)$. On the other hand, mechanical stress exceeding the decreased tensile strength of the media predisposes to within-media tearing involving a possibility of subsequent injury in intramural vasa vasorum vessels and intramural hematoma. Further, intramural hematoma has been shown to increase the stress on the affected section of the aortic wall predisposing to complete TAAD (5). However, there has been a disagreement on whether intramural hematoma is always a predecessor of actual TAAD or is it only one potential initiating factor of TAAD.

\section{Dynamic continuum of aortic disease}

During past decades, expanding literature and ongoing research have revealed numerous risk factors of TAAD involving cardiovascular diseases, connective tissue disorders, smoking, congenital and hereditary conditions, and pre-existing aortic dilatation along with several others $(4,66)$. However, either the utility or predictive power or both for most of the risk factors in a population level is low. Therefore, the ability of clinicians to identify the patients at risk of TAAD has been poor. The risk assessment has widely leaned on the assessment of the degree and the rate of aortic dilatation, existence of connective tissue disorder, and prevalence of aortic diseases among relatives of the patient (67). However, in clinical practice, also in patients with heritable disorders predisposing to aortic dissection, aortic dilatation serves as an unambiguous manifestation of the existing aortic disease (68). Since a high proportion, if not a majority of the TAAD patients, have had an ascending aorta with normal dimensions, identifying patients at risk of TAAD remains challenging (69,70). A dilemma still exists on why the ascending aorta dilates under excessive stress in some and dissects in others. As discussed earlier in this review, the degradative mechanisms of aortic wall structures and the mechanical forces, to which the wall is predisposed, are interrelated and influence one another. Thus, the process leading ultimately to TAAD is multifactorial and highly dynamic across time. A simplified causal pathway model is presented in Figure 3.

Excluding iatrogenic causes and those involving high impact blunt thoracic trauma, it is unlikely that TAAD occurs in a healthy aorta, yet the dissection requires a histopathological aortic process weakening the wall with a simultaneous stress stimulus exceeding the strength of the aortic wall. It has been shown that in aortic dilatation patients with atherosclerosis or aortitis, the aortic wall degeneration encompasses extensively the entire ascending aorta, whereas in patients without such diseases, the degeneration is focused on the aortic section affected by excessive mechanical stress-related intraluminal hemodynamic disturbances (60). In histological studies examining the content of tenascin- $\mathrm{C}$, a protein related to vascular remodeling in exposure to mechanical stress, in the media layer of patients with aortic dilatation or aortic dissection, the highest content was observed in aortic dissection patients in the dissected region (71). In specimens from patients with chronic aortic dilatation, the tenascin-C content in media was considerably lower and distributed more homogeneously around aortic media, whereas in healthy patients, tenascin-C was absent from the aortic specimens (71). Further, several histological studies have shown that dissection occurs most commonly in the outer third of the media layer in the area vascularized by the vasa vasorum network $(72,73)$. In contrast, in patients with connective tissue disorder, the dissection is located closer to the aortic lumen in the middle third of aortic media (72). Furthermore, TAAD patients with BAV have been reported to be younger, have less hypertension and more valve stenosis than those with TAV (54). These observations may suggest that TAAD in BAV patients may be more closely related to aortic fluid dynamics while in TAV patients, vascular diseases may play a more central part in the pathogenesis of TAAD. The expanding body of knowledge underlines the etiological multidimensionality involving 


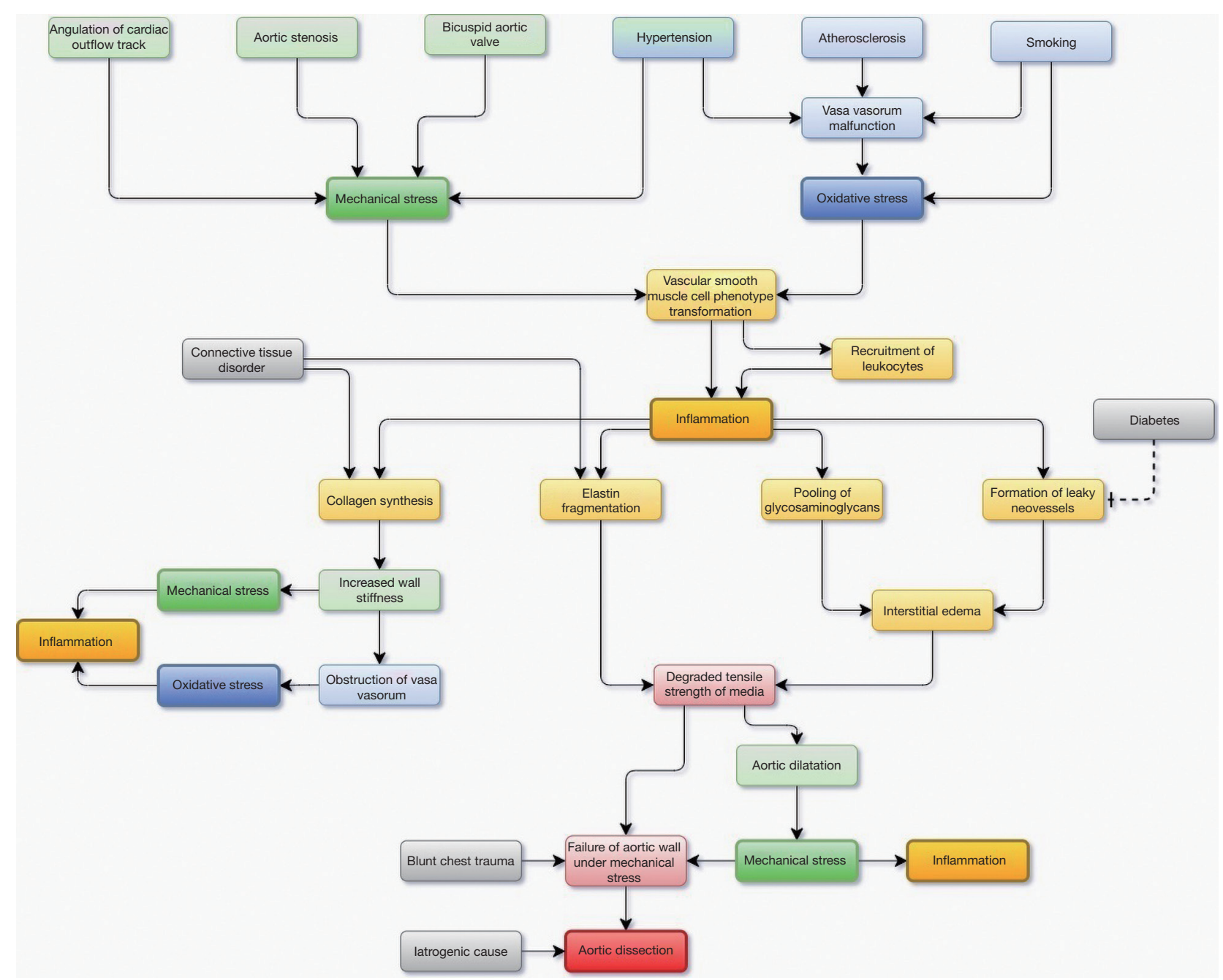

Figure 3 Causal pathway diagram on the interrelated key processes leading to aortic degeneration and type A aortic dissection (TAAD). The factors increasing mechanical stress of the aortic wall are shown in the green boxes and those increasing oxidative stress are shown in the blue boxes. The yellow boxes outline the inflammatory response in the aortic wall leading to aortic dissection.

both mechanical and oxidative stress aspects behind aortic degeneration related to TAAD.

The definitive evidence on the mechanisms determining whether the excessive stress on the aortic wall leads to aortic dilatation or TAAD is lacking. Nonetheless, based on the presented findings from the previous literature, it would seem that the pathological process leading from a healthy aorta to TAAD is gradual with a highly dynamic aspect over time and progressing aortic degeneration. The ability of the aorta to conform with the stress under which it is exposed derives from an equilibrium of the strength and the healing capacity of aortic wall structures and the intensity of the stress. This equilibrium is related to the mechanical and histological factors discussed earlier in this review. Indeed, it has been reported that the thickness of the media layer decreases along with the increasing diameter of aorta in patients with dissection of the dilated aorta but not in patients with dilated aorta but no dissection (70). These findings support the gradual nature of equilibrium of the strength and remodeling capacity in relation to mechanical stress in the aortic wall.

The continuum from a healthy aorta to a complete dissection may be conceptualized in a two-dimensional model with three steps of aortic disease (Figure 4). In a normal state (A), the strength of the aortic wall exceeds the exposing mechanical forces and healing capacity is sufficient 


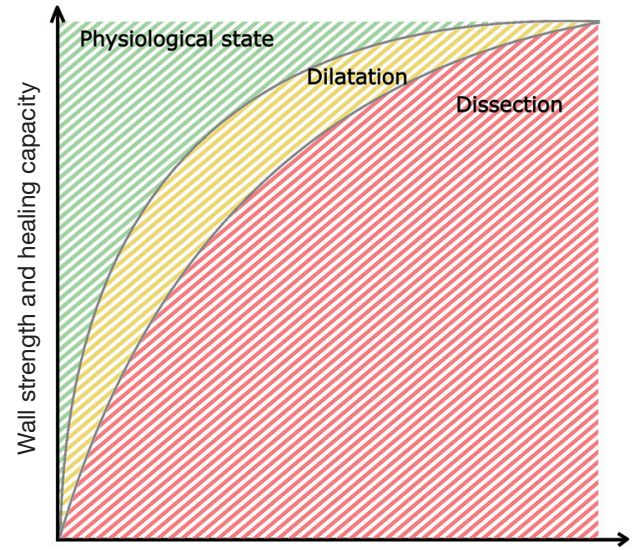

Mechanical stress intensity

Figure 4 Conceptualization of continuum of aortic disease leading to type A aortic dissection (TAAD). The $\mathrm{x}$-axis represents the intensity of mechanical stress towards the aortic wall and the $y$-axis represents the strength and healing capacity of the aortic wall under mechanical stress. In a physiological state (green area), the capability of the aortic wall to respond to the stress is sufficient to maintain the histological structure. When mechanical stress increases or the healing capacity decreases (yellow area), the ability of the aortic wall to maintain the structure impairs leading to gradual aortic dilatation. Aortic dissection occurs when mechanical impact exceeds the cohesive strength of aortic layers (red area).

to maintain the wall structure. When the stress increases or the healing capacity decreases, the ability of the aortic wall to maintain the structure impairs (B). In a chronic condition, the strength of the aortic wall decreases leading to the inability to resist the intraluminal pressure during the constant healing process. As a result, the aortic diameter increases leading to dilatation. A complete TAAD occurs, when the force against the aortic wall exceeds the wall strength leading to rupture of the intima and inner media layer leading to infiltration of blood flow between the aortic layers and formation of false lumen (C). The culmination of aortic disease into TAAD may occur via an occasional increase in mechanical stress, such as during strong physical exertion, or via a gradual failure of wall strength due to chronic inflammation. In the latter, the development of complete TAAD is initiated when the gradually degraded wall strength is overcome by the conventional mechanical stress.

\section{Summary}

In summary, the pathophysiological process leading ultimately to aortic dissection is regulated by several distinct yet interrelated physical and biochemical factors. The risk of TAAD is not a linear but rather a dynamic phenomenon. Although some of the risk factors are cumulative and progressive, others may vary by time. The relativity between the factors influencing aortic wall strength and healing capacity and factors influencing mechanical stress on the aortic wall should be considered in the risk assessment. Accounting for the dynamical property of the aortic disease in assessing the need for preventive surgical aortic reconstruction may provide a wider perspective in identifying patients at risk of TAAD and in planning preventive medical therapies, such as those against hypertension and hypercholesterolemia. Further, knowledge on the pathological process behind TAAD may provide insight into potential outcomes after preventive surgery as well as into the healing potential of the diseased aorta after reconstruction. Despite the shortcomings of the relatively simplified model, the presented concept provides a framework in the overall risk assessment of patients with aortic disease. Still, much is to be learned from the process leading to aortic dissection, especially from early warning signs prior to aortic dilatation or other visible consequences of the progressed disease. Tools for identifying the patients at risk of TAAD are needed in clinical practice.

\section{Acknowledgments}

The author is deeply grateful for professor Ville M. Mattila for covering the article processing fee.

Funding: None.

\section{Footnote}

Reporting Checklist: The author has completed the Narrative Review reporting checklist. Available at https://dx.doi. org/10.21037/jtd-21-829

Peer Review File: Available at https://dx.doi.org/10.21037/ jtd-21-829

Conflicts of Interest: The author has completed the ICMJE uniform disclosure form (available at https://dx.doi. org/10.21037/jtd-21-829). The author has no conflicts of interest to declare.

Ethical Statement: The author is accountable for all 
aspects of the work in ensuring that questions related to the accuracy or integrity of any part of the work are appropriately investigated and resolved.

Open Access Statement: This is an Open Access article distributed in accordance with the Creative Commons Attribution-NonCommercial-NoDerivs 4.0 International License (CC BY-NC-ND 4.0), which permits the noncommercial replication and distribution of the article with the strict proviso that no changes or edits are made and the original work is properly cited (including links to both the formal publication through the relevant DOI and the license). See: https://creativecommons.org/licenses/by-nc-nd/4.0/.

\section{References}

1. Nienaber CA, Clough RE. Management of acute aortic dissection. Lancet 2015;385:800-11.

2. Jassar AS, Sundt TM 3rd. How should we manage type A aortic dissection? Gen Thorac Cardiovasc Surg 2019;67:137-45.

3. Trimarchi S, Eagle KA, Nienaber CA, et al. Role of age in acute type A aortic dissection outcome: report from the International Registry of Acute Aortic Dissection (IRAD). J Thorac Cardiovasc Surg 2010;140:784-9.

4. Nienaber CA, Powell JT. Management of acute aortic syndromes. Eur Heart J 2012;33:26-35b.

5. Khanafer K, Berguer R. Fluid-structure interaction analysis of turbulent pulsatile flow within a layered aortic wall as related to aortic dissection. J Biomech 2009;42:2642-8.

6. Azadani AN, Chitsaz S, Matthews PB, et al. Comparison of mechanical properties of human ascending aorta and aortic sinuses. Ann Thorac Surg 2012;93:87-94.

7. Silva C, Reis AH. Structure and adaptation of arteries to pulsatile flow: the case of the ascending aorta. Med Phys 2014;41:063701.

8. Aquaro GD, Briatico Vangosa A, Toia P, et al. Aortic elasticity indices by magnetic resonance predict progression of ascending aorta dilation. Eur Radiol 2017;27:1395-403.

9. Hohri $Y$, Numata $S$, Itatani K, et al. Prediction for future occurrence of type A aortic dissection using computational fluid dynamics. Eur J Cardiothorac Surg 2021;60:384-91.

10. Lansac E, Lim HS, Shomura Y, et al. Aortic root dynamics are asymmetric. J Heart Valve Dis 2005;14:400-7.

11. Tonar Z, Kubíková T, Prior C, et al. Segmental and age differences in the elastin network, collagen, and smooth muscle phenotype in the tunica media of the porcine aorta.
Ann Anat 2015;201:79-90.

12. Wu D, Shen YH, Russell L, et al. Molecular mechanisms of thoracic aortic dissection. J Surg Res 2013;184:907-24.

13. Tonar $Z$, Tomášek $P$, Loskot $P$, et al. Vasa vasorum in the tunica media and tunica adventitia of the porcine aorta. Ann Anat 2016;205:22-36.

14. Sano M, Unno N, Sasaki T, et al. Topologic distributions of vasa vasorum and lymphatic vasa vasorum in the aortic adventitia--Implications for the prevalence of aortic diseases. Atherosclerosis 2016;247:127-34.

15. Baikoussis NG, Apostolakis EE, Papakonstantinou NA, et al. The implication of vasa vasorum in surgical diseases of the aorta. Eur J Cardiothorac Surg 2011;40:412-7.

16. Federspiel JM, Tschernig T, Laschke MW, et al. The vasa vasorum reach deep into the human thoracic aorta. Ann Anat 2019;225:54-6.

17. Belcaro G, Laurora G, Cesarone MR, et al. Vasa vasorum visualised by power-Doppler in normal and arteriosclerotic carotid arteries. Vasa 1996;25:226-32.

18. Scotland RS, Vallance PJ, Ahluwalia A. Endogenous factors involved in regulation of tone of arterial vasa vasorum: implications for conduit vessel physiology. Cardiovasc Res 2000;46:403-11.

19. El-Hamamsy I, Yacoub MH. Cellular and molecular mechanisms of thoracic aortic aneurysms. Nat Rev Cardiol 2009;6:771-86.

20. Lim S, Park S. Role of vascular smooth muscle cell in the inflammation of atherosclerosis. BMB Rep 2014;47:1-7.

21. Howard DP, Sideso E, Handa A, et al. Incidence, risk factors, outcome and projected future burden of acute aortic dissection. Ann Cardiothorac Surg 2014;3:278-84.

22. Hagan PG, Nienaber CA, Isselbacher EM, et al. The International Registry of Acute Aortic Dissection (IRAD): new insights into an old disease. JAMA 2000;283:897-903.

23. Landenhed M, Engström G, Gottsäter A, et al. Risk profiles for aortic dissection and ruptured or surgically treated aneurysms: a prospective cohort study. J Am Heart Assoc 2015;4:e001513.

24. Jia LX, Zhang WM, Zhang HJ, et al. Mechanical stretchinduced endoplasmic reticulum stress, apoptosis and inflammation contribute to thoracic aortic aneurysm and dissection. J Pathol 2015;236:373-83.

25. Stegemann JP, Hong H, Nerem RM. Mechanical, biochemical, and extracellular matrix effects on vascular smooth muscle cell phenotype. J Appl Physiol (1985) 2005;98:2321-7.

26. Tanaka H, Unno N, Suzuki Y, et al. Hypoperfusion of the Aortic Wall Secondary to Degeneration of Adventitial Vasa 
Vasorum Causes Abdominal Aortic Aneurysms. Curr Drug Targets 2018;19:1327-32.

27. Kakafika AI, Mikhailidis DP. Smoking and aortic diseases. Circ J 2007;71:1173-80.

28. Virdis A, Giannarelli C, Neves MF, et al. Cigarette smoking and hypertension. Curr Pharm Des 2010;16:2518-25.

29. Müller BT, Modlich O, Prisack HB, et al. Gene expression profiles in the acutely dissected human aorta. Eur J Vasc Endovasc Surg 2002;24:356-64.

30. Deplano V, Boufi M, Gariboldi V, et al. Mechanical characterisation of human ascending aorta dissection. J Biomech 2019;94:138-46.

31. Wu J, Montaniel KR, Saleh MA, et al. Origin of MatrixProducing Cells That Contribute to Aortic Fibrosis in Hypertension. Hypertension 2016;67:461-8.

32. Angouras D, Sokolis DP, Dosios T, et al. Effect of impaired vasa vasorum flow on the structure and mechanics of the thoracic aorta: implications for the pathogenesis of aortic dissection. Eur J Cardiothorac Surg 2000;17:468-73.

33. Kessler K, Borges LF, Ho-Tin-Noé B, et al. Angiogenesis and remodelling in human thoracic aortic aneurysms. Cardiovasc Res 2014;104:147-59.

34. Mallat Z, Tedgui A, Henrion D. Role of Microvascular Tone and Extracellular Matrix Contraction in the Regulation of Interstitial Fluid: Implications for Aortic Dissection. Arterioscler Thromb Vasc Biol 2016;36:1742-7.

35. Veerman KJ, Venegas-Pino DE, Shi Y, et al. Hyperglycaemia is associated with impaired vasa vasorum neovascularization and accelerated atherosclerosis in apolipoprotein-E deficient mice. Atherosclerosis 2013;227:250-8.

36. Avdic T, Franzén S, Zarrouk M, et al. Reduced Long-Term Risk of Aortic Aneurysm and Aortic Dissection Among Individuals With Type 2 Diabetes Mellitus: A Nationwide Observational Study. J Am Heart Assoc 2018;7:007618.

37. Takagi H, Umemoto T; ALICE (All-Literature Investigation of Cardiovascular Evidence) Group. Negative Association of Diabetes With Thoracic Aortic Dissection and Aneurysm. Angiology 2017;68:216-24.

38. He X, Liu X, Liu W, et al. Association between Diabetes and Risk of Aortic Dissection: A Case-Control Study in a Chinese Population. PLoS One 2015;10:e0142697.

39. Reed RK, Lidén A, Rubin K. Edema and fluid dynamics in connective tissue remodelling. J Mol Cell Cardiol 2010;48:518-23.

40. Reed RK, Rubin K. Transcapillary exchange: role and importance of the interstitial fluid pressure and the extracellular matrix. Cardiovasc Res 2010;87:211-7.

41. Roccabianca S, Ateshian GA, Humphrey JD.

Biomechanical roles of medial pooling of glycosaminoglycans in thoracic aortic dissection. Biomech Model Mechanobiol 2014;13:13-25.

42. Sugawara J, Hayashi K, Yokoi T, et al. Age-associated elongation of the ascending aorta in adults. JACC Cardiovasc Imaging 2008;1:739-48.

43. Costantino S, Paneni F, Cosentino F. Ageing, metabolism and cardiovascular disease. J Physiol 2016;594:2061-73.

44. Samani NJ, van der Harst P. Biological ageing and cardiovascular disease. Heart 2008;94:537-9.

45. Ferrucci L, Fabbri E. Inflammageing: chronic inflammation in ageing, cardiovascular disease, and frailty. Nat Rev Cardiol 2018;15:505-22.

46. Haskett D, Johnson G, Zhou A, et al. Microstructural and biomechanical alterations of the human aorta as a function of age and location. Biomech Model Mechanobiol 2010;9:725-36.

47. Fritze O, Romero B, Schleicher M, et al. Age-related changes in the elastic tissue of the human aorta. J Vasc Res 2012;49:77-86.

48. Martin C, Sun W, Primiano C, et al. Age-dependent ascending aorta mechanics assessed through multiphase CT. Ann Biomed Eng 2013;41:2565-74.

49. Yang F, Wang D, Liu H, et al. Analysis of elasticity characteristics of ascending aorta, descending aorta and pulmonary artery using 640 slice-volume CT. Medicine (Baltimore) 2018;97:e11125.

50. Wang L, Wang J, Xie M, et al. Clinical study of the ascending aorta wall motion by velocity vector imaging in patients with primary hypertension. J Huazhong Univ Sci Technolog Med Sci 2009;29:127-30.

51. Vergara C, Viscardi F, Antiga L, et al. Influence of bicuspid valve geometry on ascending aortic fluid dynamics: a parametric study. Artif Organs 2012;36:368-78.

52. Viscardi F, Vergara C, Antiga L, et al. Comparative finite element model analysis of ascending aortic flow in bicuspid and tricuspid aortic valve. Artif Organs 2010;34:1114-20.

53. Russo CF, Cannata A, Lanfranconi M, et al. Is aortic wall degeneration related to bicuspid aortic valve anatomy in patients with valvular disease? J Thorac Cardiovasc Surg 2008;136:937-42.

54. Eleid MF, Forde I, Edwards WD, et al. Type A aortic dissection in patients with bicuspid aortic valves: clinical and pathological comparison with tricuspid aortic valves. Heart 2013;99:1668-74.

55. Kauhanen SP, Liimatainen T, Kariniemi E, et al. A 
smaller heart-aorta-angle associates with ascending aortic dilatation and increases wall shear stress. Eur Radiol 2020;30:5149-57.

56. Hickson SS, Butlin M, Graves M, et al. The relationship of age with regional aortic stiffness and diameter. JACC Cardiovasc Imaging 2010;3:1247-55.

57. Wallace SM, Yasmin, McEniery CM, et al. Isolated systolic hypertension is characterized by increased aortic stiffness and endothelial dysfunction. Hypertension 2007;50:228-33.

58. Swinne CJ, Shapiro EP, Jamart J, et al. Age-associated changes in left ventricular outflow tract geometry in normal subjects. Am J Cardiol 1996;78:1070-3.

59. D'ostrevy N, Ngo H, Magnin B, et al. Bicuspid and tricuspid aortic valve do not have the same ascending aorta morphology. Clin Anat 2018;31:693-7.

60. Agozzino L, Santè P, Ferraraccio F, et al. Ascending aorta dilatation in aortic valve disease: morphological analysis of medial changes. Heart Vessels 2006;21:213-20.

61. Youssefi P, Gomez A, He T, et al. Patient-specific computational fluid dynamics-assessment of aortic hemodynamics in a spectrum of aortic valve pathologies. J Thorac Cardiovasc Surg 2017;153:8-20.e3.

62. Plonek T, Zak M, Rylski B, et al. Wall stress correlates with intimal entry tear localization in Type A aortic dissection. Interact Cardiovasc Thorac Surg 2018;27:797-801.

63. Numata S, Itatani K, Kanda K, et al. Blood flow analysis of the aortic arch using computational fluid dynamics. Eur J Cardiothorac Surg 2016;49:1578-85.

64. Gao F, Watanabe M, Matsuzawa T. Stress analysis in a layered aortic arch model under pulsatile blood flow. Biomed Eng Online 2006;5:25.

Cite this article as: Uimonen M. Synthesis of multidimensional pathophysiological process leading to type A aortic dissection: a narrative review. J Thorac Dis 2021;13(10):6026-6036. doi: $10.21037 /$ jtd-21-829
65. Thubrikar MJ, Agali P, Robicsek F. Wall stress as a possible mechanism for the development of transverse intimal tears in aortic dissections. J Med Eng Technol 1999;23:127-34.

66. Gawinecka J, Schönrath F, von Eckardstein A. Acute aortic dissection: pathogenesis, risk factors and diagnosis. Swiss Med Wkly 2017;147:w14489.

67. Davies RR, Goldstein LJ, Coady MA, et al. Yearly rupture or dissection rates for thoracic aortic aneurysms: simple prediction based on size. Ann Thorac Surg 2002;73:17-27; discussion 27-8.

68. Gleason TG. Heritable disorders predisposing to aortic dissection. Semin Thorac Cardiovasc Surg 2005;17:274-81.

69. Krüger T, Sandoval Boburg R, Lescan M, et al. Aortic elongation in aortic aneurysm and dissection: the Tübingen Aortic Pathoanatomy (TAIPAN) project. Eur J Cardiothorac Surg 2018;54:26-33.

70. Van Puyvelde J, Verbeken E, Verbrugghe P, et al. Aortic wall thickness in patients with ascending aortic aneurysm versus acute aortic dissection. Eur J Cardiothorac Surg 2016;49:756-62.

71. Trescher K, Thometich B, Demyanets S, et al. Type A dissection and chronic dilatation: tenascin-C as a key factor in destabilization of the aortic wall. Interact Cardiovasc Thorac Surg 2013;17:365-70.

72. Osada H, Kyogoku M, Matsuo T, et al. Histopathological evaluation of aortic dissection: a comparison of congenital versus acquired aortic wall weakness. Interact Cardiovasc Thorac Surg 2018;27:277-83.

73. Grewal N, Velders BJJ, Gittenberger-de Groot AC, et al. A Systematic Histopathologic Evaluation of Type-A Aortic Dissections Implies a Uniform Multiple-Hit Causation. J Cardiovasc Dev Dis 2021;8:12. 